\title{
Gesellschaftliche Bereiche des freiwilligen Engagements
}

\author{
Corinna Kausmann \& Christine Hagen
}

\section{Kernaussagen}

Freiwilliges Engagement wird am häufigsten in den Bereichen Sport und Bewegung, Kultur und Musik sowie im sozialen Bereich ausgeübt. 13,5 Prozent der Menschen $\mathrm{ab} 14$ Jahren übernehmen freiwillige Arbeiten oder Aufgaben im Bereich Sport und Bewegung. Im Engagementfeld Kultur und Musik, im sozialen Bereich sowie auch im Bereich Schule und Kindergarten engagieren sich jeweils gut acht Prozent der Menschen in Deutschland.

Der größte Zuwachs an freiwillig Engagierten zwischen 2014 und 2019 ist in dem Bereich Umwelt, Naturschutz oder Tierschutz auszumachen. Berücksichtigt man die Entwicklung in den letzten zwanzig Jahren von 1999 bis 2019, sind die vier Bereiche mit den höchsten Anteilen Engagierter auch die Bereiche mit dem größten Wachstum (sozialer Bereich, Kultur und Musik, Sport und Bewegung, Schule und Kindergarten).

In den Bereichen Schule und Kindergarten sowie Sport und Bewegung gibt es die größten Unterschiede zwischen engagierten Frauen und Männern. Frauen engagieren sich häufiger als Männer in familienbezogenen und sozialen Bereichen. Männer sind häufiger in den Bereichen Sport und Bewegung, Unfall- oder Rettungsdienste oder freiwillige Feuerwehr und auch im Bereich der Politik und der politischen Interessenvertretung freiwillig tätig. 
Das freiwillige Engagement in den gesellschaftlichen Bereichen ist geprägt durch altersspezifische Unterschiede. Insbesondere im sportlichen Bereich sowie in der außerschulischen Jugendarbeit und Bildungsarbeit für Erwachsene und im Unfall-, Rettungsdienst oder der freiwilligen Feuerwehr dominiert anteilig das Engagement der Jüngeren. Menschen ab 50 Jahren sind hingegen im sozialen Bereich anteilig häufiger engagiert als Personen unter 50 Jahren. 


\subsection{Einleitung}

Das freiwillige Engagement in Deutschland wird in den verschiedensten gesellschaftlichen Bereichen ausgeübt. Diese reichen von Sport, Kultur oder Freizeit über Politik und Soziales bis zu Umwelt und Tierschutz. Die verschiedenen Engagementbereiche erfahren durch gesellschaftliche Entwicklungen und sich wandelnde Rahmenbedingungen unterschiedlichen Zulauf. Im Deutschen Freiwilligensurvey werden vierzehn gesellschaftliche Bereiche unterschieden, in denen sich Menschen freiwillig engagieren. Diese Bereiche werden seit der ersten Erhebung des Freiwilligensurveys im Jahr 1999 abgefragt. Sie orientieren sich an der Abfrage zum freiwilligen Engagement aus dem Speyerer Wertesurvey von 1997 (von Rosenbladt 2001: 39). In der ersten Erhebung des Freiwilligensurveys waren noch fünfzehn gesellschaftliche Bereiche Bestandteil der Abfrage. Der Bereich der wirtschaftlichen Selbsthilfe wurde aber aufgrund der geringen Beteiligungsquote in den folgenden Befragungen nicht mehr erfasst. Zwischen den vierzehn Engagementbereichen im Freiwilligensurvey finden sich deutliche Unterschiede in Hinblick darauf, wie stark die Beteiligung an den jeweiligen Bereichen ausgeprägt ist. Die bisherigen Befragungen zeigen, dass sich anteilig die meisten Menschen im Bereich Sport und Bewegung engagieren. Ebenfalls ist eine hohe Beteiligung in den Engagementbereichen Schule oder Kindergarten, Kultur und Musik, im sozialen Bereich sowie im kirchlichen oder religiösen Bereich zu finden. Die geringsten Anteile freiwillig Engagierter waren für den Bereich Justiz oder Kriminalitätsprobleme, für den Gesundheitsbereich oder auch für den Bereich der beruflichen Interessenvertretung auszumachen (Vogel et al. 2017).

Die Entscheidung, sich in einem bestimmten Bereich zu engagieren, ist sowohl durch individuelle Interessen als auch durch Gelegenheitsstrukturen geleitet. Die Verteilung des Engagements auf die Bereiche kann gerade im Zeitvergleich auch gesellschaftliche Trends abbilden. Zunahmen oder Abnahmen der Engagementbeteiligung in bestimmten Bereichen können beispielsweise durch eine veränderte Wahrnehmung gesellschaftlicher Herausforderungen beeinflusst werden. Die Veränderungen der Beteiligung in den Engagementbereichen können durch den Freiwilligensurvey seit 1999 abgebildet werden. Betrachtet man die Engagementbeteiligung in den Bereichen im Trend, konnten 2014 Wachstumsbereiche ausgemacht werden: In den Bereichen Schule und Kindergarten, Kultur und Musik, im sozialen Bereich sowie in der Jugendarbeit und Bildungsarbeit für Erwachsene gab es seit 1999 einen deutlichen Anstieg des Engagements (Vogel et al. 2017). Mit dem Freiwilligensurvey 2019 wird untersucht, ob sich die Trends fortsetzen oder ob andere Entwicklungen oder thematische Verlagerungen zu beobachten sind. Vor dem Hintergrund der gestiegenen öffentlichen Thematisierung der Klimakrise in den letzten Jahren wäre zu erwarten, dass sich dies auch in einem Aufschwung des freiwilligen Engagements im Bereich Umwelt, Natur- und Tierschutz niederschlägt. So ist beispielsweise 2019 mit der Bewegung Fridays for Future das Thema Umwelt politisch und medial in den Fokus der Öffentlichkeit gerückt. Die klimatischen Bedingungen sind eine der großen Her- 
ausforderungen für Politik und Gesellschaft und die Fragen zum Klimaschutz und zur Energiewende motivieren viele - vor allem junge Menschen - zum Engagement (Deutscher Bundestag 2017: 443 ff.).

Die klimatischen Veränderungen könnten aber auch auf andere Bereiche des freiwilligen Engagements, zum Beispiel den Bereich Unfall- oder Rettungsdienste oder freiwillige Feuerwehr einen Einfluss haben. So führt die Zunahme extremer Wetterereignisse - wie Starkregen, Stürme oder lange Hitze und Trockenheit - zu neuen Umweltgefahren in Deutschland, die wiederum mit einem erhöhten Einsatzbedarf von Rettungsdiensten verbunden sind. Viele Hilfs- und Rettungsorganisationen werden in erster Linie - zumindest in ländlichen Regionen - durch freiwillig Engagierte getragen. Möglicherweise ergibt sich durch einen erhöhten Einsatzbedarf, dass sich mehr Personen in diesem Bereich engagieren. Wiederum kann die Abwanderung aus ländlichen Regionen - insbesondere von jüngeren Menschen - eher zu einem geringeren Engagement in diesem Bereich führen (Deutscher Bundestag 2017: $451 \mathrm{ff}$.).

Weiterhin können sich Veränderungen auf der institutionellen und organisatorischen Ebene auf die Beteiligung in den Engagementbereichen auswirken (Alscher et al. 2021). So hat beispielsweise das eher kurzfristige oder kurzzeitige Engagement in den letzten Jahren an Bedeutung gewonnen. Einige Engagementbereiche - auch die mit eher formal organisierten Engagementstrukturen - können sich dieser Entwicklung anpassen und versuchen sie zu nutzen. So wurden beim Deutschen Roten Kreuz (DRK) zum Beispiel Personen für die Koordination ausgebildet, die diese ungebundene spontane Hilfe vor Ort in die bestehenden formell organisierten Strukturen einbinden (Deutscher Bundestag 2017: 457 f.; Neufeind et al. 2015).

Doch nicht nur gesellschaftliche Veränderungen und organisatorische Rahmenbedingungen haben einen Einfluss auf die gesellschaftlichen Bereiche, in denen sich Menschen freiwillig engagieren. Auch die familiären Strukturen, Geschlechterrollen sowie altersspezifische Interessen sind ausschlaggebend, wie sowohl die Ergebnisse des letzten Freiwilligensurveys als auch die Ergebnisse der letzten Zeitverwendungserhebung zeigen (Burkhardt et al. 2017; Kausmann et al. 2017; Vogel et al. 2017). So kann sich die immer noch zu beobachtende gesellschaftliche Arbeitsteilung der Geschlechter auch in den gesellschaftlichen Bereichen des Engagements widerspiegeln. Auch die lebensphasenspezifischen Anknüpfungspunkte beispielsweise über die Freizeitbeschäftigung, den Beruf, die schulische Ausbildung oder familiäre Aufgaben können mit der Beteiligung in den verschiedenen Engagementbereichen in Zusammenhang stehen. In diesem Kapitel sollen folgende Fragen beantwortet werden:

a) In welchen Bereichen sind die Menschen 2019 freiwillig engagiert?

b) Hat das freiwillige Engagement in den letzten zwanzig Jahren in den einzelnen gesellschaftlichen Bereichen zu- oder abgenommen?

c) $\mathrm{Zu}$ welchen Anteilen engagieren sich Frauen und Männer und welche Unterschiede zwischen den Altersgruppen gibt es in den Engagementbereichen? 


\subsection{Daten und Indikatoren}

Die Ergebnisse des vorliegenden Kapitels beruhen auf den Daten des Deutschen Freiwilligensurveys (FWS) und werden gewichtet dargestellt. Die Gewichtung zielt darauf ab, die Repräsentativität zu verbessern, um verallgemeinerbare Aussagen treffen zu können. In den Veröffentlichungen zu den Erhebungen der Jahre 1999 bis 2014 wurden im Rahmen des Freiwilligensurveys bislang die Gewichtungsmerkmale Geschlecht, Alter, Bundesland sowie Gemeindegrößenklasse herangezogen. Diese Gewichtung wird nun um das Gewichtungsmerkmal der schulischen Bildung ergänzt. Personen aus unterschiedlichen Bildungsgruppen nehmen mit unterschiedlicher Wahrscheinlichkeit an Umfragen teil; daher sind verschiedene Bildungsgruppen in den Daten über- beziehungsweise unterrepräsentiert. Die Gewichtung inklusive schulischer Bildung wird in diesem Bericht auf die Daten aller fünf Erhebungswellen von 1999 bis 2019 angewendet. Die Engagementquoten fallen durch dieses Vorgehen für alle Erhebungswellen niedriger aus als bisher berichtet; es ändert sich somit das Niveau, der Trend bleibt jedoch im Wesentlichen bestehen. Die Gewichtung der Daten inklusive Bildung wirkt sich auch auf andere Ergebnisse aus.

Alle Ergebnisse werden auf statistische Signifikanz getestet (verwendet wird ein Signifikanzniveau von $\mathrm{p}<0,05)$. Die Prüfung auf statistische Signifikanz zeigt an, ob ein anhand der Daten des Freiwilligensurveys gefundener Unterschied mit großer Wahrscheinlichkeit auch in der Grundgesamtheit, also beispielsweise in der Wohnbevölkerung Deutschlands, vorhanden ist (statistisch signifikant) oder nur zufällig in den Daten vorliegt (statistisch nicht signifikant).

Die dargestellten Ergebnisse fußen auf der Befragung des Freiwilligensurveys 2019, die von März bis November 2019 erfolgte. Somit ist es mithilfe der Daten des Freiwilligensurveys 2019 nicht möglich, Aussagen über spätere Entwicklungen wie beispielsweise die Auswirkungen der Corona-Pandemie auf das freiwillige Engagement zu tätigen.

Die zweistufige Abfrage zum freiwilligen Engagement ist verbunden mit der Erfassung unterschiedlicher gesellschaftlicher Bereiche, in denen sich die Menschen aktiv beteiligen: Für die Ermittlung dieser Aktivitäten werden in der Befragung vierzehn Gesellschaftsbereiche benannt, in denen man mitmachen oder sich aktiv beteiligen kann. Die Abfrage lautet:

Es gibt vielfältige Möglichkeiten, außerhalb von Beruf und Familie irgendwo mitzumachen, beispielsweise in einem Verein, einer Initiative, einem Projekt oder einer Selbsthilfegruppe. Ich nenne Ihnen verschiedene Bereiche, die dafür in Frage kommen. Wenn Sie an die letzten 12 Monate denken: Haben Sie sich in einem oder mehreren dieser Bereiche aktiv beteiligt? Sind Sie oder waren Sie irgendwo aktiv...

(1) ... im Bereich Sport und Bewegung

(2) ... im Bereich Kultur und Musik

(3) ... im Bereich Freizeit und Geselligkeit 
(4) ... im sozialen Bereich

(5) ... im Gesundheitsbereich

(6) ... im Bereich Schule oder Kindergarten

(7) ... in der außerschulischen Jugendarbeit oder der Bildungsarbeit für Erwachsene

(8) ... im Bereich Umwelt, Naturschutz oder Tierschutz

(9) ... im Bereich Politik und politische Interessenvertretung

(10) ... im Bereich der beruflichen Interessenvertretung außerhalb des Betriebes

(11) ... im kirchlichen oder religiösen Bereich

(12) ... im Bereich der Justiz und der Kriminalitätsprobleme

(13) ... im Unfall- oder Rettungsdienst oder in der freiwilligen Feuerwehr

(14) ... in einem bisher noch nicht genannten Bereich

Mehrfachnennungen möglich; Antwortkategorien (jeweils): ja; nein

Das freiwillige Engagement wird im Freiwilligensurvey im zweiten Schritt dieses Verfahrens ermittelt. Dazu bekommen Personen, die in der Abfrage zur Aktivität in mindestens einem der Bereiche angegeben haben, sich aktiv zu beteiligen, diese Folgefrage gestellt:

Uns interessiert nun, ob Sie in den Bereichen, in denen Sie aktiv sind, auch ehrenamtliche Tätigkeiten ausüben oder in Vereinen, Initiativen, Projekten oder Selbsthilfegruppen engagiert sind. Es geht um freiwillig übernommene Aufgaben und Arbeiten, die man unbezahlt oder gegen geringe Aufwandsentschädigung ausübt.

Sie sagten, Sie sind im Bereich [Name des jeweiligen Bereichs] aktiv. Wenn Sie an die letzten 12 Monate denken: Haben Sie in diesem Bereich auch Aufgaben oder Arbeiten übernommen, die Sie freiwillig oder ehrenamtlich ausüben?

Antwortkategorien (jeweils): ja; nein

Die Befragten können in mehreren unterschiedlichen Bereichen freiwillig engagiert sein sowie mehrere freiwillige Tätigkeiten in einem Bereich ausüben. In der Erfassung des freiwilligen Engagements haben sie auch die Möglichkeit, dies anzugeben. Die Angaben zum freiwilligen Engagement in den vierzehn gesellschaftlichen Bereichen umfassen somit Mehrfachnennungen. Für jeden Bereich, in dem sich die Befragten sowohl aktiv beteiligen als auch freiwillig engagieren, werden die freiwilligen Tätigkeiten anhand offener Abfragen detaillierter erfasst. Dazu werden der organisatorische Rahmen, die ausgeübten Aufgaben und der Name der Tätigkeit erfasst (siehe auch Kapitel 2). Beispiele für diese offenen Angaben der Befragten zu den einzelnen freiwilligen Tätigkeiten sind in den Bereichsprofilen (Abschnitt 5.4) dargestellt. Die Bereichsprofile umfassen ebenfalls Angaben zu der Engagementbeteiligung in dem jeweiligen Bereich im Trend sowie für 2019 differenziert nach Geschlecht und Altersgruppen. Die verwendeten Differenzierungsmerkmale werden in Kapitel 2 beschrieben. 


\subsection{Gesellschaftliche Bereiche des freiwilligen Engagements}

Der gesellschaftliche Bereich, in dem sich die meisten Menschen freiwillig engagieren, ist der Bereich Sport und Bewegung. 13,5 Prozent der Menschen ab 14 Jahren übernehmen freiwillige Arbeiten oder Aufgaben in diesem Bereich (Abbildung 5-1). Auch für Kultur und Musik (8,6 Prozent), im sozialen Bereich (8,3 Prozent) oder im Bereich Schule und Kindergarten (8,2 Prozent) engagieren sich die Menschen in Deutschland anteilig besonders häufig. $\mathrm{Zu}$ etwas geringeren Anteilen betätigen sie sich im kirchlichen oder religiösen Bereich mit 6,8 Prozent oder im Bereich der Freizeit und Geselligkeit mit 6,1 Prozent. Die Engagementbeteiligung für Umwelt, Natur-

Abbildung 5-1 Anteile freiwillig engagierter Personen in vierzehn Bereichen 2019 (Basis: alle Befragten; Mehrfachnennungen möglich)

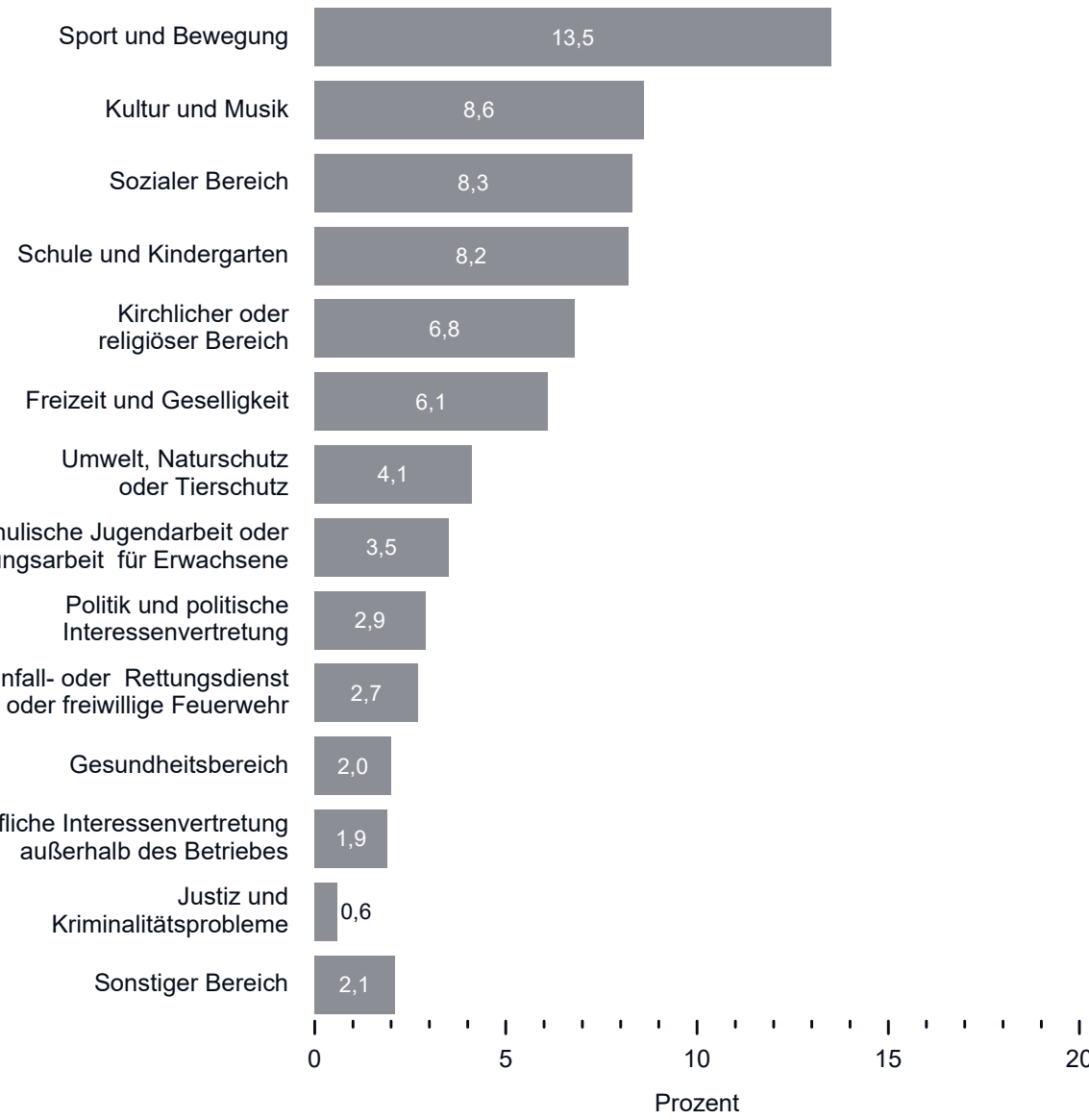

Quelle: FWS 2019 gewichtet, eigene Berechnungen (DZA). Basis: alle Befragten ( $n=27.762)$. Mehrfachnennungen möglich. 
schutz und den Tierschutz beträgt 4,1 Prozent. Die Engagementquoten in den Bereichen der außerschulischen Jugendarbeit oder Bildungsarbeit für Erwachsene, in der Politik oder politischen Interessenvertretung sowie im Unfall- oder Rettungsdient beziehungsweise in der freiwilligen Feuerwehr liegen zwischen 2,7 und 3,5 Prozent. Die geringsten Engagementquoten weisen der Gesundheitsbereich, der Bereich der beruflichen Interessenvertretung außerhalb des Betriebes sowie der Bereich der Justiz und Kriminalitätsprobleme auf.

In Deutschland sind im Jahr 2019 mehr als die Hälfte (51,1 Prozent) der freiwillig Engagierten mehrfach engagiert, das bedeutet, sie üben zwei oder mehr freiwillige Tätigkeiten aus. Im Freiwilligensurvey wird für die Mehrfachengagierten erfasst, welche ihrer freiwilligen Tätigkeiten die zeitaufwendigste ist. Wenn für alle Befragten nur eine freiwillige Tätigkeit (die einzige bei Einfachengagierten, die zeitaufwendigste bei Mehrfachengagierten) betrachtet wird, zeigt sich eine ganz ähnliche Verteilung in den Bereichen (ohne Abbildung) wie bei der Betrachtung des gesamten Engagements - bei dem alle Bereiche berücksichtigt werden, in denen eine Person tätig ist.

\subsection{Profile der gesellschaftlichen Bereiche}

In den folgenden Abschnitten werden die dreizehn thematischen Engagementbereiche einzeln vorgestellt. Der Bereich Sonstiges wird nicht dargestellt. Die Bereichsprofile weisen die Anteile der freiwillig Engagierten für alle fünf durchgeführten Befragungen seit 1999 auf, sodass die Entwicklung der letzten zwanzig Jahre in den jeweiligen Engagementbereichen nachgezeichnet werden kann. Neben den Gesamtanteilen in Prozent werden auch hochgerechnete Angaben der Personen in Millionen für die Beteiligung in den Engagementbereichen ausgegeben. Für die Hochrechnungen werden dieselben Kriterien hinzugezogen, wie für die prozentuale Gewichtung der Daten (Bundesland, Gemeindegrößenklassen (BIK), Geschlecht, Altersgruppe, schulische Bildung). Des Weiteren werden in den Profilen die Anteile von freiwillig Engagierten nach Geschlecht sowie die Anteile der Engagierten unterschiedlicher Altersgruppen für die jeweiligen Bereiche im Jahr 2019 dargestellt. Die Befragten können in mehreren Bereichen freiwillig engagiert sein, somit umfassen alle Bereichsprofile Mehrfachnennungen.

Um eine Vorstellung über die konkreten Aufgaben und Arbeiten im freiwilligen Engagement in den unterschiedlichen Bereichen zu bekommen, werden in den Profilen jeweils Beispiele für die Angaben von Tätigkeiten der Engagierten genannt. Diese Beispiele wurden wortgleich aus den offenen Tätigkeitsangaben der Befragten entnommen. Im Freiwilligensurvey 2019 liegen 25.242 Tätigkeitsangaben vor, die über Inhalt und organisatorische Anbindung der freiwilligen Tätigkeiten Aufschluss geben. Diese Angaben ermöglichen einen Überblick über die vielfältigen Tätigkeitsfelder und -inhalte der freiwillig Engagierten in Deutschland. Darüber hinaus dienten diese 
Angaben im Rahmen der Datenaufbereitung unter Zuhilfenahme eines Kriterienkataloges der Prüfung, inwiefern es sich bei der jeweiligen Tätigkeit um freiwilliges Engagement handelt (siehe Kapitel 2). Alle dargestellten Beispiele in den Profilen sind als freiwillige Tätigkeiten gewertet worden. Eine Prüfung der Bereichszuordnung hat nicht stattgefunden, die Zuordnung basiert auf der Einschätzung der Befragten.

\subsubsection{Sport und Bewegung}

Im Jahr 2019 sind 13,5 Prozent der Menschen in Deutschland im Bereich Sport und Bewegung freiwillig engagiert (Abbildung 5-2). Während sich die Anteile der Engagierten zwischen 1999 und 2004 in diesem Bereich kaum verändert haben, ist der Anteil von 10,2 Prozent im Jahr 2004 auf 9,o Prozent im Jahr 2009 gesunken und dann

Abbildung 5-2 Anteile engagierter Personen im Bereich Sport und Bewegung (Basis: alle Befragten)

Im Zeitvergleich

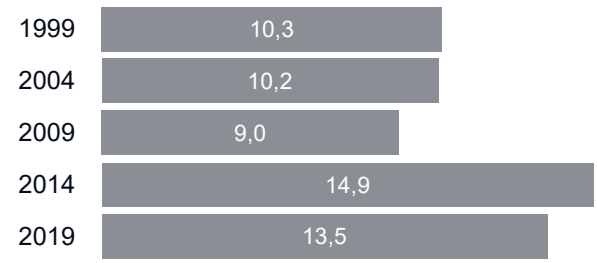

Anteile engagierter Personen 2019

Frauen

Männer

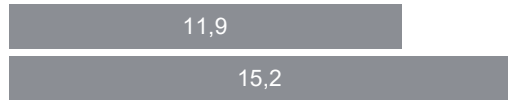

14-29 Jahre

30-49 Jahre

50-64 Jahre

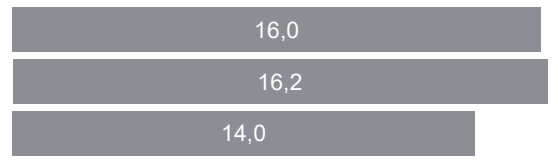

65 Jahre und älter

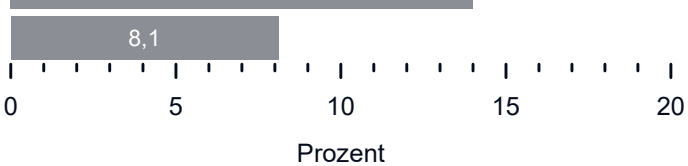

Quelle: FWS, gewichtet, eigene Berechnungen (DZA). Basis: alle Befragten. FWS $1999(n=14.917)$, FWS 2004 ( $n=15.000)$, FWS 2009 $(\mathrm{n}=20.003)$, FWS $2014(\mathrm{n}=28.690)$, FWS $2019(\mathrm{n}=27.762)$.

Es gibt keinen statistisch signifikanten Unterschied zwischen 1999 und 2004. 2009, 2014 sowie 2019 unterscheiden sich jeweils statistisch signifikant zu allen Jahren. Der Geschlechterunterschied ist statistisch signifikant. Bei den Altersgruppen ist nur der Unterschied zwischen den 14- bis 29-Jährigen und den 30- bis 49-Jährigen nicht statistisch signifikant. 
auf 14,9 Prozent im Jahr 2014 angestiegen. Von 2014 bis 2019 gibt es einen leichten Rückgang in der Beteiligung um 1,4 Prozentpunkte. Insgesamt engagieren sich 2019 rund 9,8 Millionen Menschen im Bereich Sport und Bewegung. 1999 waren es rund 7,2 Millionen.

Auffällig ist, dass sich im Sportbereich anteilig mehr Männer als Frauen engagieren und dass es anteilig insbesondere die jüngeren Altersgruppen sind, die in diesem Bereich engagiert sind. 16, o Prozent der 14- bis 29-Jährigen sowie 16,2 Prozent der 30bis 49-Jährigen engagieren sich im Bereich Sport und Bewegung. Im Vergleich sind diese Anteile doppelt so hoch wie die Anteile freiwillig Engagierter bei den 65-Jährigen und Älteren in diesem Bereich.

Der organisatorische Rahmen für freiwilliges Engagement im Sportbereich ist ganz überwiegend der Verein. Das spiegeln auch die Beispiele der Tätigkeiten wider. Neben der Leitung von Übungs- und Trainingseinheiten, werden hier auch viele andere Tätigkeiten, wie das Organisieren von Veranstaltungen oder koordinierende Aufgaben innerhalb des Vereinslebens genannt.

Beispiele für Tätigkeiten der Engagierten im Bereich Sport und Bewegung im FWS 2019:

- Turniertanzverein: Organisation von Wettkämpfen

- Sportverein: Kassiererin

- Turnverein: Übungsleiterin für Geräteturnen

- Handballverein: Schiedsrichter

- Schwimmverein: Rettungsschwimmer und Ausbilder

- Altenverein: Turnleiter, Wandern mit Senioren

- Fußballverein: Zeugwart, Hilfe für Flüchtlinge

- Tischtennisverein: Mannschaftsführer, Organisation der Spiele

- Hockeyclub: Datenschutzbeauftragter

- Sportverein: Organisation von Festen und Veranstaltungen

- Sportclub: Betreuung einer Jugendmannschaft und in der Redaktion einer Mitgliedszeitschrift

- Kampfsporttrainer: Jungendkoordinator und Übungsleiter

\subsubsection{Kultur und Musik}

Die Engagementquote beträgt 2019 im Bereich Kultur und Musik 8,6 Prozent. Der Anteil Engagierter ist somit etwa doppelt so hoch wie vor zwanzig Jahren und auch im Vergleich zu 2014 ist die Engagementquote 2019 leicht angestiegen (Abbildung 5-3). 1999 waren rund 2,9 Millionen Personen ab 14 Jahren in diesem Bereich engagiert, bis 2019 ist die Zahl auf rund 6,3 Millionen angewachsen.

Hervorzuheben ist, dass im Bereich Kultur und Musik kein statistisch signifikanter Unterschied in der Beteiligung von Frauen und Männern auszumachen ist. 
Abbildung 5-3 Anteile engagierter Personen im Bereich Kultur und Musik (Basis: alle Befragten)

Im Zeitvergleich

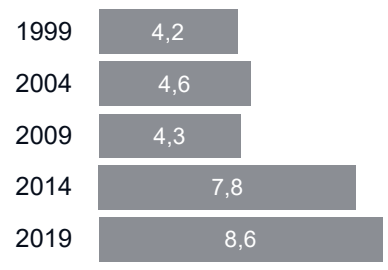

Anteile engagierter Personen 2019

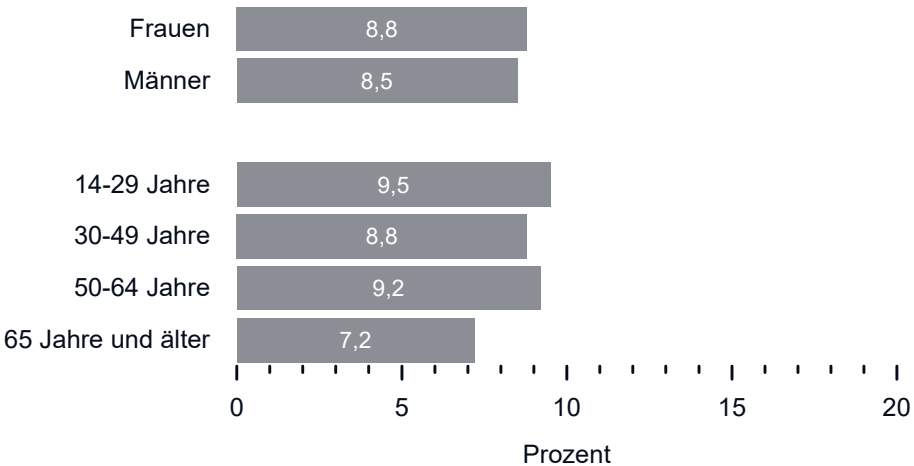

Quelle: FWS, gewichtet, eigene Berechnungen (DZA). Basis: alle Befragten. FWS 1999 ( $n=14.915)$, FWS 2004 ( $n=15.000)$, FWS 2009 $(n=20.002)$, FWS $2014(n=28.690)$, FWS 2019 ( $n=27.762)$.

Die Trendunterschiede sind statistisch signifikant, außer die Unterschiede zwischen 1999, 2004 und 2009. Der Geschlechterunterschied ist nicht statistisch signifikant. Im Vergleich der Altersgruppen ist nur der Unterschied zwischen den 65-Jährigen und Älteren zu den anderen drei Gruppen statistisch signifikant.

8,8 Prozent der Frauen und 8,5 Prozent der Männer sind in diesem Bereich freiwillig engagiert, zum Beispiel in einer Theater- oder Musikgruppe, in einem Chor, einer kulturellen Vereinigung oder in einem Förderverein. Für die Altersgruppen sind ebenfalls nur geringfügige Unterschiede auszumachen (Abbildung 5-3): Während sich die Anteile freiwilligen Engagements im Bereich Kultur und Musik zwischen den drei jüngeren Altersgruppen ( 14 bis 29 Jahre, 30 bis 49 Jahre, 50 bis 64 Jahre) statistisch nicht signifikant voneinander unterscheiden, ist der Anteil der 65-Jährigen und Älteren mit 7,2 Prozent etwas geringer.

Beispiele für Tätigkeiten der Engagierten im Bereich Kultur und Musik im FWS 2019:

- Chor: Erster Chorvorsitzender

- Verein für interkulturellen Austausch: Erwachsenenbildung

- Förderverein Kunsthalle: Führungen, Kunstpate

- Theatergruppe: Kassenwartin 
- Religiöser Kulturverein: organisatorische Aufgaben (Sekretär)

- Förderverein vom Planetarium: Kassenprüfer

- Volkstanzgruppe: Tanzleiterin

- Öffentliche Bücherei: Schriftführer und Verantwortlicher für die IT

- Orchester: Gestaltung der Website

- Museum: Restaurierung von technischen Geräten

\subsubsection{Sozialer Bereich}

Das Engagement im sozialen Bereich kann beispielsweise freiwillige Tätigkeiten in einem Wohlfahrtsverband oder einer anderen Hilfsorganisation, in der Nachbarschaftshilfe oder in einer Selbsthilfegruppe umfassen. 1999 übernehmen 3,4 Prozent der Menschen in Deutschland ein freiwilliges Engagement in diesem Bereich. Die Engagementquote steigt seitdem an - mit Ausnahme von $2004 \mathrm{zu} 2009$ - und beträgt im Jahr 2019 8,3 Prozent. Insgesamt engagierten sich 1999 rund 2,4 Millionen Menschen ab 14 Jahren im sozialen Bereich, 2019 ist die Zahl auf rund 6,o Millionen angestiegen und damit mehr als doppelt so hoch wie vor zwanzig Jahren.

Frauen sind anteilig häufiger im sozialen Bereich engagiert als Männer; die Differenz der Engagementquoten beträgt 2,o Prozentpunkte (Abbildung 5-4). Anteilig besonders häufig ist die Altersgruppe von 50 bis 64 Jahren mit 10,2 Prozent in diesem Bereich freiwillig engagiert. Daran schließen sich mit 8,8 Prozent die 65-Jährigen und Älteren an. Die Anteile der freiwillig Engagierten im Alter von 14 bis 29 Jahren und im Alter von 30 bis 49 Jahren unterscheiden sich nicht statistisch signifikant.

Beispiele für Tätigkeiten der Engagierten im sozialen Bereich im FWS 2019:

- Tafel: Ausgabe von Lebensmitteln

- Stadtrat: Vorsitzender des Sozialausschusses

- Flüchtlingshilfe: Koordinator der Deutschklassen

- Wohlfahrtsverband (Caritas): Nachhilfelehrerin für Migranten

- Nachbarschaftshilfe für Senioren: Besuche und Hilfsdienste

- Behindertenwerkstatt: Organisation von Festen, Elternbeirat

- Beratung für Migranten: Sprachlehrerin, Beraterin

- Kinderschutz: Kassenwartin

- Selbsthilfegruppe zur Trauerbewältigung: Organisation von Wanderungen

- Wohlfahrtsverband (AWO): Alltagsassistenz für ältere Menschen 
Abbildung 5-4 Anteile engagierter Personen im sozialen Bereich (Basis: alle Befragten) Im Zeitvergleich

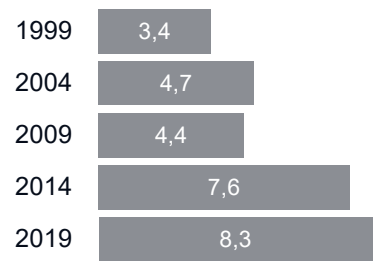

Anteile engagierter Personen 2019
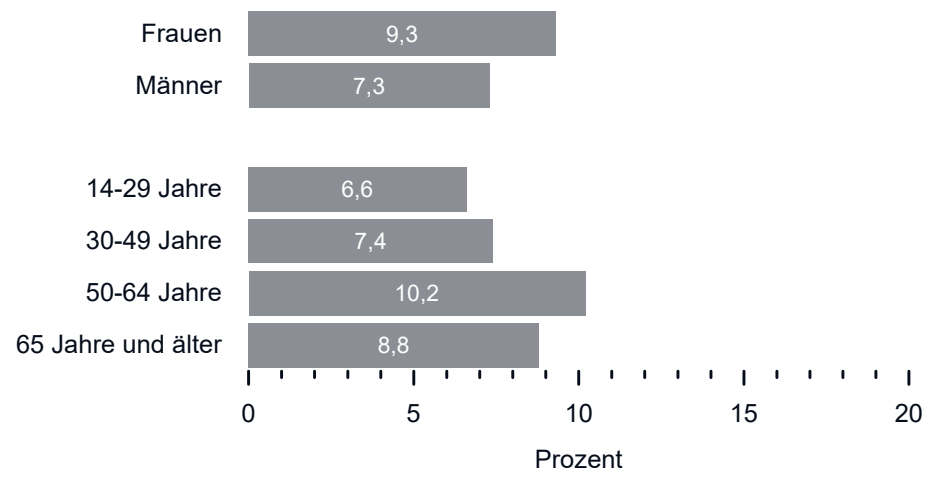

Quelle: FWS, gewichtet, eigene Berechnungen (DZA). Basis: alle Befragten. FWS 1999 ( $n=14.914)$, FWS 2004 ( $n=15.000)$, FWS 2009 ( $n=19.997)$, FWS 2014 ( $n=28.690)$, FWS $2019(n=27.762)$.

Die Trendunterschiede sind statistisch signifikant, außer der Unterschied zwischen 2004 und 2009. Sowohl die Geschlechterunterschiede als auch die Unterschiede nach Altersgruppen sind statistisch signifikant, mit Ausnahme des Unterschieds zwischen den 14- bis 29-Jährigen und den 30 - bis 49-Jährigen.

\subsubsection{Schule und Kindergarten}

2019 engagieren sich 8,2 Prozent der Menschen im Bereich Schule und Kindergarten. Auf einem ähnlich hohen Niveau war auch die Beteiligung im Jahr 2014 mit 8,1 Prozent. Im Trend der letzten zwanzig Jahre gab es jedoch einen deutlichen Anstieg von 3,2 Prozentpunkten (Abbildung 5-5). Das entspricht einer Anzahl von rund 3,5 Millionen Menschen 1999 und rund 5,9 Millionen Menschen 2019, die sich im Bereich der Schule oder des Kindergartens engagieren.

Besonders große Unterschiede zeigen sich in diesem Bereich zwischen Frauen und Männern. 10,2 Prozent der Frauen und 6,1 Prozent der Männer in Deutschland engagieren sich im Jahr 2019 in der Schule oder im Kindergarten, zum Beispiel in der Elternvertretung, der Schülervertretung oder in einem Förderverein. Auch bei den Altersgruppen sind große Unterschiede zu beobachten: 17,3 Prozent der Menschen im Alter von 30 bis 49 Jahren engagieren sich freiwillig in der Schule oder im Kindergarten. Dieser Anteil ist mehr als doppelt so hoch wie der Anteil in der jüngsten Al- 
Abbildung 5-5 Anteile engagierter Personen im Bereich Schule und Kindergarten (Basis: alle Befragten)

Im Zeitvergleich

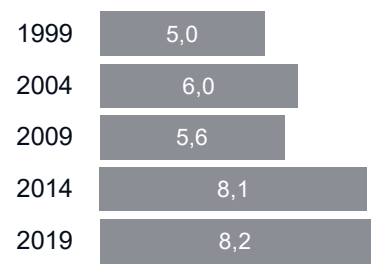

Anteile engagierter Personen 2019

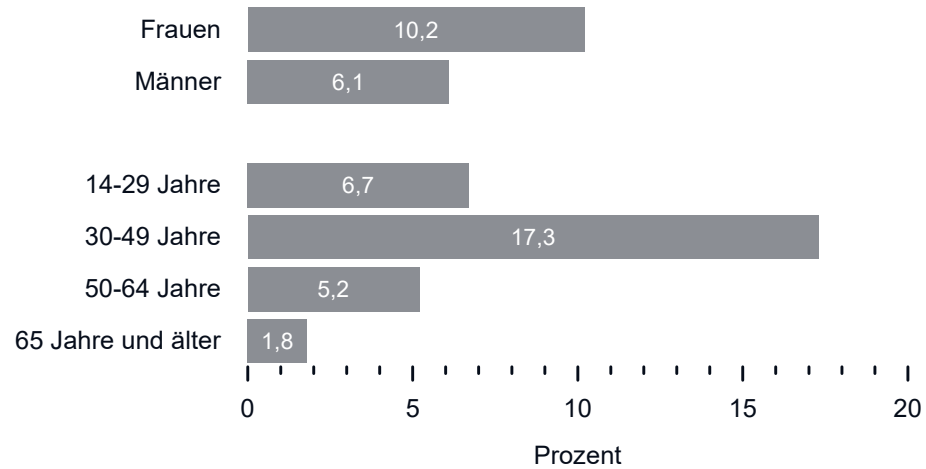

Quelle: FWS, gewichtet, eigene Berechnungen (DZA). Basis: alle Befragten. FWS 1999 ( $n=14.917)$, FWS 2004 ( $n=15.000$ ), FWS 2009 $(n=20.002)$, FWS $2014(n=28.690)$, FWS 2019 ( $n=27.762)$.

Die Trendunterschiede sind statistisch signifikant, mit Ausnahme der Jahre 2004 und 2009 sowie 2014 und 2019. Sowohl die Geschlechterunterschiede als auch die Unterschiede nach Altersgruppen sind statistisch signifikant.

tersgruppe (14 bis 29 Jahre: 6,7 Prozent) oder der Anteil der Menschen im Alter von 50 bis 64 Jahren (5,2 Prozent). Bei den Menschen im Alter von 65 Jahren und älter ist der Anteil geringer (1,8 Prozent).

Beispiele für Tätigkeiten der Engagierten im Bereich Schule und Kindergarten im FWS 2019:

- Ferienlager: Kinder- und Jugendbetreuer

- Grundschule: AG-Leiter für Holzwerken

- Schule: Elternvertretung

- Schülervertretung: Organisation von Schulfesten

- Kindergarten: Elternvertreterin und Kassenwartin

- Grundschule: Hausaufgabenhilfe

- Örtliches Nachhilfeinstitut: ehrenamtliche Nachhilfelehrerin

- Kindergarten: Lesepate

- Schule: Streitschlichter für Schüler 
- Förderverein in Grundschule: Rechnungsprüfer

- Grundschule und Kindergarten: Brandschutzerziehung der freiwilligen Feuerwehr

- Kindergarten: Kursleiterin für musikalische Früherziehung

\subsubsection{Kirchlicher oder religiöser Bereich}

Das freiwillige Engagement im Kontext von Kirche und Religion ist von 1999 bis 2014 gestiegen. Während sich 1999 4,5 Prozent der Menschen in diesem Bereich engagieren, waren es 2004 5,2 Prozent und 2014 bereits 6,8 Prozent. In den beiden Befragungen 2014 und 2019 ist der Anteil Engagierter mit 6,8 Prozent - rund 4,9 Millionen Menschen - konstant geblieben. 1999 waren es rund 3,2 Millionen Menschen, die sich im kirchlichen oder religiösen Bereich engagierten.

Abbildung 5-6 Anteile engagierter Personen im kirchlichen oder religiösen Bereich (Basis: alle Befragten)

Im Zeitvergleich

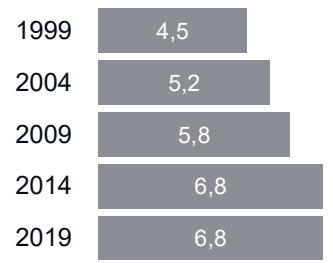

Anteile engagierter Personen 2019

Frauen

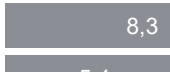

Männer

5,4

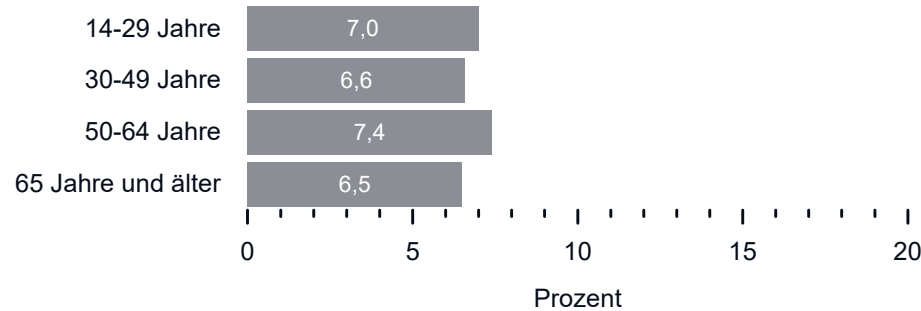

Quelle: FWS, gewichtet, eigene Berechnungen (DZA). Basis: alle Befragten. FWS 1999 ( $n=14.919)$, FWS $2004(n=15.000)$, FWS 2009 $(n=20.001)$, FWS $2014(n=28.690)$, FWS $2019(n=27.762)$.

Die Trendunterschiede sind statistisch signifikant, bis auf die Unterschiede zwischen 2004 und 2009 sowie zwischen 2014 und 2019 . Während die Geschlechterunterschiede statistisch signifikant sind, finden sich zwischen den Altersgruppen keine statistisch signifikanten Unterschiede. 
8,3 Prozent der Frauen engagieren sich 2019 im kirchlichen oder religiösen Bereich. Bei den Männern ist der Anteil geringer und beträgt 5,4 Prozent. Herausstechend für diesen gesellschaftlichen Bereich des freiwilligen Engagements ist im Vergleich zu allen anderen gesellschaftlichen Bereichen, dass sich keinerlei statistisch signifikante Unterschiede zwischen den Altersgruppen zeigen (Abbildung 5-6).

Beispiele für Tätigkeiten der Engagierten im kirchlichen oder religiösen Bereich im FWS 2019:

- Gemeinde/Kirchenrat: Vorbereitung der Gottesdienste, Kinder- und Jugendarbeit

- Kirchengemeinde: Kirchenbuchführerin

- Kirchengemeinde: Begleitung bei Freizeitaktivitäten

- Katholische Arbeitnehmerbewegung: Kreisvorsitzender

- Katholische Kirche: Lektor, Kantor

- Meditationsgruppe Buddhismus: Meditationsleitung

- Kirchlicher Bauausschuss: Bauprojekte begleiten, Pastoren beraten

- Kirchliche Seelsorge

- Obdachlosenbetreuung: Koordination der Gruppe, Kochen für Obdachlose

- Moschee: Kinderbetreuung und Integrationshilfe

- Jüdische Gemeinde: Leitung der Vertreterversammlung

- Islamischer Kulturverein: Technische Unterstützung und Betreuung

\subsubsection{Freizeit und Geselligkeit}

Das Engagement im Bereich Freizeit und Geselligkeit findet beispielsweise in Kleingartenvereinen oder im Rahmen von Spieletreffs statt. Dieser Bereich ist einer von den wenigen hier dargestellten Bereichen, in denen im Trend nur geringfügige Unterschiede in der Engagementbeteiligung in den letzten zwanzig Jahren auszumachen sind. Die Engagementquote lag im Jahr 2009 bei 4,5 Prozent und ist damit statistisch signifikant niedriger als in allen anderen Jahren (Abbildung 5-7). 2019 liegt die Beteiligung bei 6,1 Prozent. Insgesamt engagieren sich 2019 rund 4,4 Millionen Menschen im Bereich Freizeit und Geselligkeit. Im Jahr 1999 waren es rund 3,9 Millionen Menschen.

Männer engagieren sich anteilig häufiger (6,7 Prozent) im Bereich Freizeit und Geselligkeit als Frauen (5,5 Prozent). Beim Vergleich der Altersgruppen sind die jeweiligen Anteile der Engagierten sehr ähnlich. Es gibt keinen statistisch signifikanten Unterschied zwischen den vier Altersgruppen.

Beispiele für Tätigkeiten der Engagierten im Bereich Freizeit und Geselligkeit im FWS 2019:

- Flugmodellsportverein: allgemeine Hilfen (wie Rasenmähen)

- Dorfverein: Zweite Vereinsvorsitzende (Versammlungen und Feste organisieren) 
- Gartenverein: Verantwortlich für Elektroanlage

- Karnevalsclub: Programm gestalten

- Pfadfinder: Betreuer von Kindergruppen

- Blindenverein: Moderatorin verschiedener Veranstaltungen

- Landfrauen: Rabattenpflege im Ort, Organisation von Sommerfesten und etwas basteln

- Organisation eines Nachbarschaftstreffens

- Europäische Städtepartnerschaft: Tätigkeit in 2 Komitees

- Imkerverein: Schriftführern, 1. Vorsitz

Abbildung 5-7 Anteile engagierter Personen im Bereich Freizeit und Geselligkeit (Basis: alle Befragten)

Im Zeitvergleich

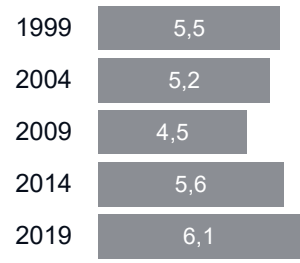

Anteile engagierter Personen 2019

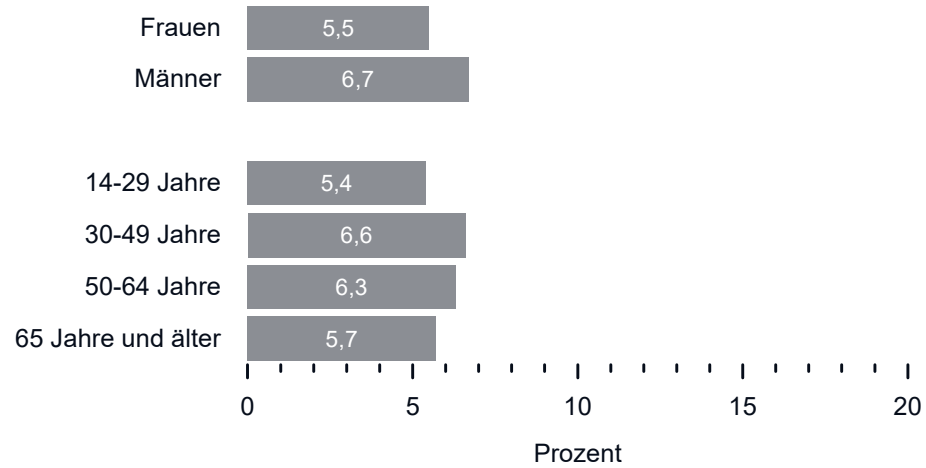

Quelle: FWS, gewichtet, eigene Berechnungen (DZA). Basis: alle Befragten. FWS 1999 ( $n=14.906)$, FWS 2004 ( $n=15.000)$, FWS 2009 $(n=19.993)$, FWS 2014 ( $n=28.690)$, FWS $2019(n=27.762)$.

Die Engagementquote im Jahr 2009 unterscheidet sich statistisch signifikant zu allen anderen Erhebungsjahren. Auch 2004 und 2019 unterscheiden sich statistisch signifikant voneinander. Die anderen Trendunterschiede sind nicht statistisch signifikant. Der Unterschied zwischen den Geschlechtern ist statistisch signifikant. Es gibt keine statistisch signifikanten Unterschiede zwischen den Altersgruppen. 


\subsubsection{Umwelt, Naturschutz oder Tierschutz}

Das freiwillige Engagement für Umwelt, Natur- und Tierschutz ist in den letzten zwanzig Jahren deutlich gestiegen (Abbildung 5-8). Während sich 1999 noch 1,6 Prozent der Menschen in Deutschland für Umwelt, Natur- oder Tierschutz engagierten, sind es im Jahr 2019 4,1 Prozent. Zwischen 2014 und 2019 ist eine Zunahme der Engagierten um einen Prozentpunkt zu beobachten. 1999 engagierten sich in diesem Bereich rund 1,1 Millionen Menschen. Im Jahr 2019 sind es rund 2,9 Millionen Menschen, die sich für die Umwelt, den Naturschutz oder den Tierschutz freiwillig engagieren.

Frauen und Männer engagieren sich jeweils mit gleichen Anteilen (4,1 Prozent) im Bereich Umwelt, Natur- oder Tierschutz. Der höchste Anteil Engagierter in diesem Bereich findet sich unter den 50- bis 64-Jährigen mit 5,0 Prozent. In den beiden jün-

Abbildung 5-8 Anteile engagierter Personen im Bereich Umwelt, Naturschutz oder Tierschutz (Basis: alle Befragten)

Im Zeitvergleich

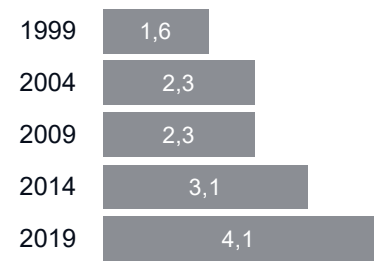

Anteile engagierter Personen 2019

Frauen

Männer

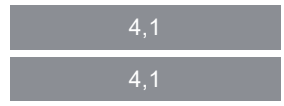

14-29 Jahre

30-49 Jahre

50-64 Jahre

65 Jahre und älter
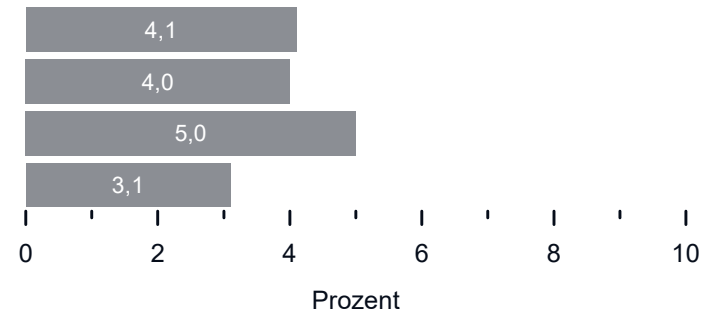

Quelle: FWS, gewichtet, eigene Berechnungen (DZA). Basis: alle Befragten. FWS $1999(n=14.919)$, FWS $2004(n=15.000)$, FWS 2009 $(n=20.002)$, FWS $2014(n=28.690)$, FWS $2019(n=27.762)$.

Die Trendunterschiede sind statistisch signifikant, bis auf die Unterschiede zwischen 2004 und 2009. Der Geschlechterunterschied ist nicht statistisch signifikant. Die Unterschiede zwischen den 30- bis 49-Jährigen, den 50- bis 64-Jährigen und den 65-Jährigen und Älteren sind statistisch signifikant. 
geren Altersgruppen ist der Anteil um rund einen Prozentpunkt geringer. Das Engagement bei den 65-Jährigen und Älteren fällt noch etwas niedriger aus.

Beispiele für Tätigkeiten der Engagierten im Bereich Umwelt, Natur- oder Tierschutz im FWS 2019:

- Naturschutzbeauftragter des Kreises

- Sportfischerverband: Kassierer, Gewässeraufsicht

- Tiernothilfe: Hilfe für ausgesetzte Tiere organisieren

- Tierheim: Hunde ausführen und Zwinger reinigen, Hilfe beim Tag der offenen Tür

- Naturschutzgruppe: Vogelzählung, Pflege von Bäumen

- Klimaschutzinitiative: Dozentin (Vorträge halten)

- Jägerschaft: Revierarbeit im Wald

- Naturschutzverein: Kassenprüfer, Hecken- und Obstbaumpflege

- Umweltschutzverein (Greenpeace): Müll sammeln

- Angelverein: Gewässerwart

- Obst- und Gartenbauverein: stellvertretende Vorsitzende im Umweltrat

- Vogelkundevereine: Schriftführer

\subsubsection{Außerschulische Jugendarbeit oder Bildungsarbeit für Erwachsene}

Die außerschulische Jugendarbeit und Bildungsarbeit für Erwachsene gehört zu den Engagementbereichen, die in den letzten zwanzig Jahren viel Zulauf erfahren haben: Von 1999 bis 2019 ist der Anteil der engagierten Menschen in diesem Bereich fast auf das Dreifache angestiegen (Abbildung 5-9). Während 1999 die Engagementquote noch bei 1,2 Prozent lag, ist sie bis 2009 auf 2,1 Prozent angestiegen und hat bis 2014 nochmal einen Zuwachs auf 3,4 Prozent erfahren. In den letzten fünf Jahren hat sich der Anteil Engagierter in diesem Bereich nicht statistisch signifikant verändert und beträgt 2019 3,5 Prozent. Über 850.00o Engagierte waren 1999 in diesem Bereich zu verzeichnen; 2019 sind es rund 2,5 Millionen Menschen, die sich für außerschulische Jugendarbeit oder Bildungsarbeit für Erwachsene engagieren. Dieser Bereich umfasst freiwillige Tätigkeiten wie das Betreuen von Kinder- oder Jugendgruppen oder das Durchführen von Bildungsveranstaltungen.

3,8 Prozent der Frauen und 3,2 Prozent der Männer in Deutschland engagieren sich in der außerschulischen Jugendarbeit oder der Bildungsarbeit für Erwachsene. Vor allem jüngere Menschen sind anteilig häufig in diesem Bereich engagiert. Über fünf Prozent der 14- bis 29-Jährigen übernehmen hier freiwillige Tätigkeiten. Bei den 65-Jährigen und Älteren sind es 2,o Prozent. 
Abbildung 5-9 Anteile engagierter Personen im Bereich der außerschulischen Jugendarbeit oder Bildungsarbeit für Erwachsene (Basis: alle Befragten)

Im Zeitvergleich

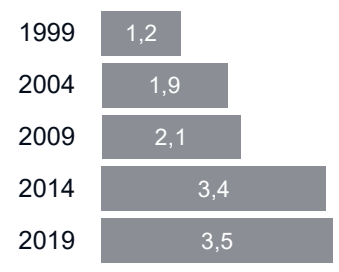

Anteile engagierter Personen 2019

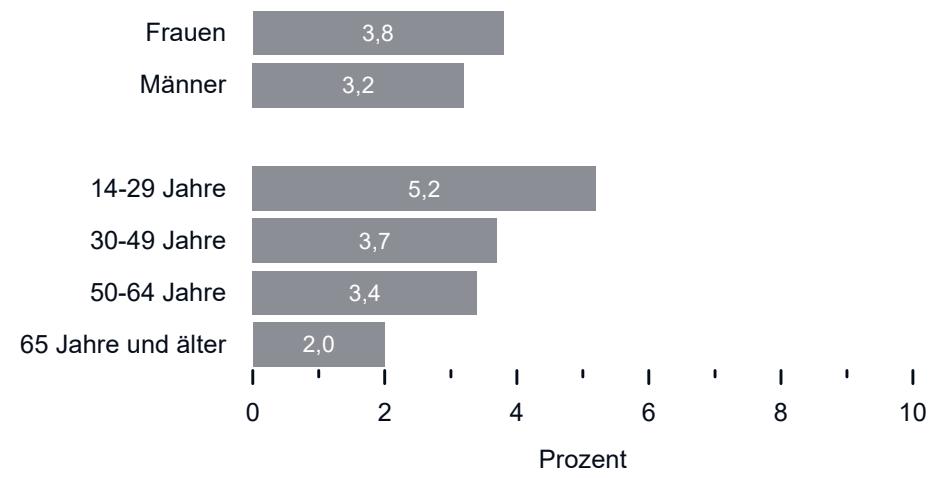

Quelle: FWS, gewichtet, eigene Berechnungen (DZA). Basis: alle Befragten. FWS 1999 ( $n=14.917)$, FWS 2004 ( $n=15.000)$, FWS 2009 $(n=20.000)$, FWS $2014(n=28.690)$, FWS $2019(n=27.762)$.

Die Unterschiede im Zeitvergleich sind statistisch signifikant, außer die Unterschiede zwischen 2004 und 2009 sowie zwischen 2014 und 2019. Die Geschlechterunterschiede sind statistisch signifikant. Im Vergleich der Altersgruppen unterscheiden sich nur die 30- bis 49-Jährigen und die 50- bis 64-Jährigen nicht statistisch signifikant voneinander.

Beispiele für Tätigkeiten der Engagierten im Bereich der außerschulischen Jugendarbeit oder Bildungsarbeit für Erwachsene im FWS 2019:

- Verein, Bildungsprojekt: Organisation und Buchhaltung

- Flüchtlingshilfe: Deutschunterricht, Patenschaft für Flüchtlinge

- Koch- und Bastelgruppe für Frauen mit Hartz IV: Kursleiterin

- Sportfischerverband: Ausbilder und Prüfer

- Gemeinnütziger Verein: Leitung der Ausbildung von SchwangerschaftskonfliktBerater/innen

- Verwaltungsakademie: ehrenamtlicher Dozent

- Kinderschutzbund: Ausbilderin für ehrenamtliche Telefonberater

- Arbeitsgemeinschaft für Erinnerungs- und Geschichtsarbeit: Vorträge, Diskussionen, Stadtführungen

- Jugendbildungsstätte: Kassierer 


\subsubsection{Politik und politische Interessenvertretung}

Insgesamt rund 2,1 Millionen Menschen ab 14 Jahren engagieren sich 2019 freiwillig in der Politik oder politischen Interessenvertretung, beispielsweise in einer Partei, im Gemeinderat oder Stadtrat, in politischen Initiativen, Solidaritätsprojekten oder Ähnlichem. 1999 betrug die Zahl der Engagierten in Deutschland in diesem Bereich rund 1,5 Millionen. Der Anteil der Menschen, die sich freiwillig im Bereich Politik und politische Interessenvertretung engagieren, hat sich in der Zeit von 1999 bis 2009 nicht statistisch signifikant verändert (Abbildung 5-10). Zwischen 2009 und 2014 ist die Engagementquote jedoch von 2,1 auf 3,o Prozent leicht gestiegen, 2019 beträgt der Anteil Engagierter in diesem Bereich 2,9 Prozent.

Der Unterschied zwischen den Geschlechtern ist im Bereich der Politik sehr deutlich. Mit einer Engagementquote von 3,9 Prozent beteiligen sich Männer anteilig

Abbildung 5-10 Anteile engagierter Personen im Bereich der Politik und politischen Interessenvertretung (Basis: alle Befragten)

Im Zeitvergleich

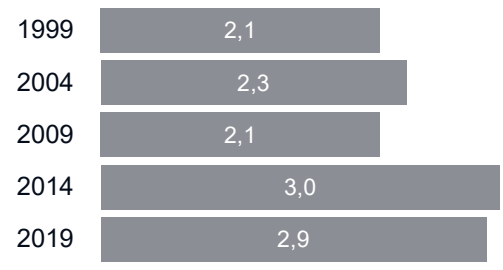

Anteile engagierter Personen 2019
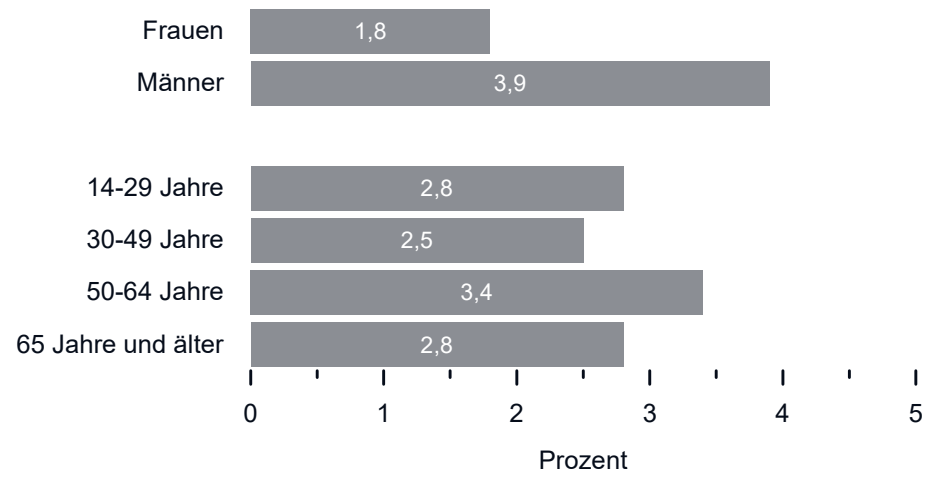

Quelle: FWS, gewichtet, eigene Berechnungen (DZA). Basis: alle Befragten. FWS 1999 ( $n=14.912)$, FWS 2004 ( $n=15.000$ ), FWS 2009 $(n=20.004)$, FWS $2014(n=28.690)$, FWS $2019(n=27.762)$.

1999, 2004 und 2009 unterscheiden sich nicht statistisch signifikant voneinander. Auch 2014 und 2019 unterscheiden sich nicht statistisch signifikant voneinander. Der Geschlechterunterschied ist statistisch signifikant. Einen statistisch signifikanten Altersunterschied gibt es nur zwischen den 30- bis 49-Jährigen und den 50- bis 64-Jährigen. 
mehr als doppelt so häufig wie Frauen, deren Engagementquote in diesem Bereich bei 1,8 Prozent liegt. Besonders Personen im Alter von 50 bis 64 Jahren engagieren sich anteilig häufig in der Politik und der politischen Interessenvertretung (3,4 Prozent).

Beispiele für Tätigkeiten der Engagierten im Bereich Politik und politische Interessenvertretung im FWS 2019:

- Partei: Leiter einer Fachkommission

- Stadtrat: Ausschussarbeit, Wahlkampf

- Gemeinderat: Ortssprecher

- Stellvertretender Bürgermeister

- Bürgerinitiative: Flugblätter verteilen, Mahnwache und Pressearbeit

- Seniorenbeirat: Organisatorische Aufgaben, Verschönerung in der Region

- Ortsgruppe von Amnesty International: Unterschriften sammeln

- Wirtschaftsrat: Wirtschaftsausschuss auf Landkreisebene

- Omas gegen rechts: Mithilfe bei dem Europafest

- Arbeitskreis Asyl: Alltagshilfe für Geflüchtete

- Fridays for Future: Demonstrationen mitorganisiert

- Politische Jugendorganisation: Organisation von Aktionstagen, Demonstrationen, Kassenwart

\subsubsection{Unfall- oder Rettungsdienst oder freiwillige Feuerwehr}

Das freiwillige Engagement in Unfall- oder Rettungsdiensten oder der freiwilligen Feuerwehr unterscheidet sich zwischen den Jahren 1999 und 2019 nicht statistisch signifikant: Jeweils rund 1,9 Millionen Menschen engagieren sich in diesem Bereich, das sind jeweils 2,7 Prozent der Personen ab 14 Jahren in Deutschland (Abbildung 5-11).

4,3 Prozent der Männer engagieren sich 2019 freiwillig in diesem Bereich. Der Anteil der Frauen, die sich in Unfall- oder Rettungsdiensten oder der freiwilligen Feuerwehr engagieren, ist mit 1,1 Prozent deutlich geringer. Solche klaren Unterschiede sind auch zwischen den Altersgruppen zu sehen. Während sich 4,4 Prozent der Personen im Alter von 14 bis 29 Jahren in diesem Bereich freiwillig engagieren und 3,4 Prozent der 30- bis 49-Jährigen, sind es bei den 50- bis 64-Jährigen 2,6 Prozent und bei den 65-Jährigen und Älteren 0,5 Prozent.

Beispiele für Tätigkeiten der Engagierten im Unfall- oder Rettungsdienst oder in der freiwilligen Feuerwehr im FWS 2019:

- Feuerwehr: Jugendwart, Übernahme der Ausbildung

- Freiwillige Feuerwehr: Feuerwehrfrau

- Deutsches Rotes Kreuz: Ersthelfereinsätze

- Technisches Hilfswerk: Ausbildungsbeauftragter 
- Wasserwacht: Rettungsschwimmer am Strand

- Rettungshundestaffel: Suche von vermissten Personen

- Technisches Hilfswerk: Koch, Verköstigung bei Einsätzen

- Freiwillige Feuerwehr: Zeugwart

- Rettungsdienst: Rettungssanitäter

- Technisches Hilfswerk: Öffentlichkeitsarbeit

- Deutsche Lebens-Rettungs-Gesellschaft (DLRG): Rettungstaucher

Abbildung 5-11 Anteile engagierter Personen im Bereich Unfall- oder Rettungsdienst oder freiwillige Feuerwehr (Basis: alle Befragten)

Im Zeitvergleich

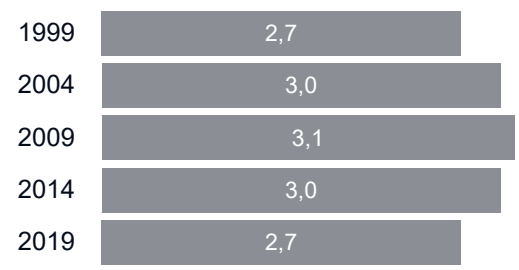

Anteile engagierter Personen 2019

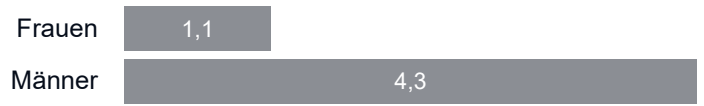

14-29 Jahre

30-49 Jahre

50-64 Jahre

65 Jahre und älter

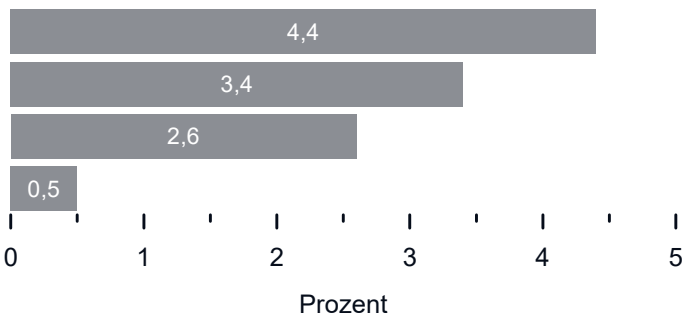

Quelle: FWS, gewichtet, eigene Berechnungen (DZA). Basis: alle Befragten. FWS 1999 ( $n=14.915)$, FWS 2004 ( $n=15.000)$, FWS 2009 $(n=20.004)$, FWS $2014(n=28.690)$, FWS $2019(n=27.762)$.

Im Zeitvergleich gibt es keine statistisch signifikanten Unterschiede. Der Geschlechterunterschied ist statistisch signifikant. Bei den Altersgruppen ist nur der Unterschied zwischen den 14- bis 29-Jährigen und den 30- bis 49-Jährigen nicht statistisch signifikant.

\subsubsection{Gesundheitsbereich}

Die Engagementquote im Gesundheitsbereich ist im Zeitraum von 1999 bis 2019 angestiegen (Abbildung 5-12). Über 680.000 Menschen engagierten sich 1999 im Gesundheitsbereich, zum Beispiel leisten sie Hilfe in der Krankenpflege, organisieren 
Abbildung 5-12 Anteile engagierter Personen im Gesundheitsbereich (Basis: alle Befragten) Im Zeitvergleich

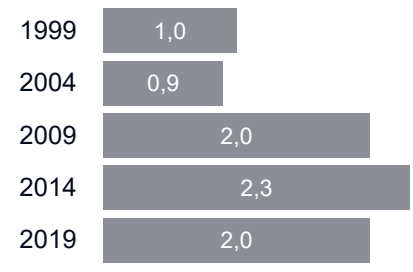

Anteile engagierter Personen 2019

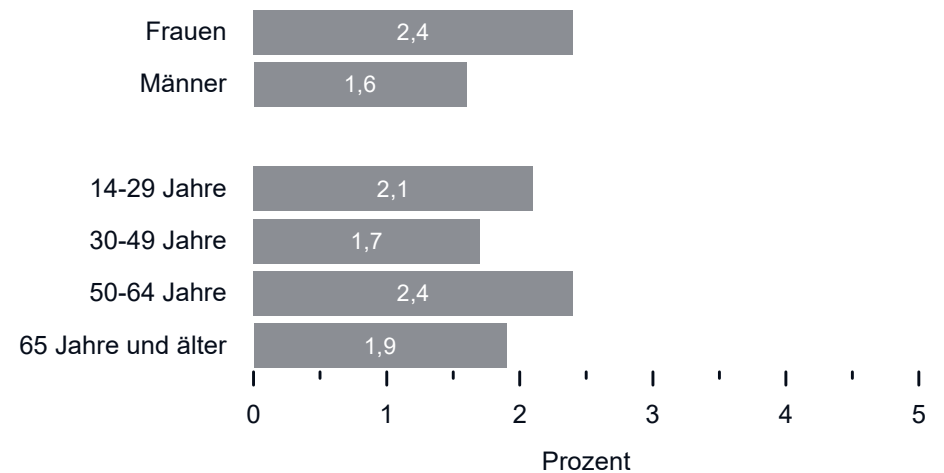

Quelle: FWS, gewichtet, eigene Berechnungen (DZA). Basis: alle Befragten. FWS $1999(n=14.915)$, FWS $2004(n=15.000)$, FWS 2009 ( $n=20.001)$, FWS 2014 ( $n=28.690)$, FWS $2019(n=27.762)$.

Der Unterschied im Zeitvergleich zwischen 1999 und 2004 ist nicht statistisch signifikant. Auch die Unterschiede zwischen 2009,2014 und 2019 sind nicht statistisch signifikant. Der Geschlechterunterschied ist statistisch signifikant. Bei den Altersgruppen ist nur der Unterschied zwischen den 30 - bis 49-Jährigen und den 50 - bis 64-Jährigen statistisch signifikant.

oder machen Besuchsdienste oder engagieren sich in einer Selbsthilfegruppe. Im Jahr 2019 sind es rund 1,4 Millionen Menschen.

Mit 2,4 Prozent engagieren sich Frauen anteilig häufiger im Gesundheitsbereich als Männer mit 1,6 Prozent. Im Vergleich der Altersgruppen sind die Unterschiede eher gering. Bei den Menschen im Alter von 50 bis 64 Jahren ist der Anteil am höchsten und liegt bei 2,4 Prozent.

Beispiele für Tätigkeiten der Engagierten im Gesundheitsbereich im FWS 2019:

- Hospizhilfe: Betreuung der Betroffenen und Angehörigen

- Psychosoziales Zentrum: Klangmassage und systemische Beratung

- Wohlfahrtsverband (Medizinisches Katastrophen-Hilfswerk Deutschland): Sanitäter

- Ehrenamtlicher Betreuungsverein: Betreuung von Menschen mit Behinderung

- Mobiler Pflegedienst: Helferin beim Einkaufen oder bei Arztbesuchen 
- Deutsche Kinderkrebsstiftung: Mentor, Unterstützung und Besuch von Kindern

- Wohlfahrtsverband (Deutsches Rotes Kreuz): Psychosoziale Notfallversorgung

- Pflegeheim: ehrenamtlicher Helfer

- Ernährungsberatung: Vorträge und Seminare für Eltern, Erzieher und im Altenheim

- Grüne Dame: Betreuung von Kranken in Krankenhäusern und Altenheimen

- Selbsthilfegruppe für Tumorerkrankte: Gruppenleiter

- Wohlfahrtsverband (Deutsches Rotes Kreuz): Rettungseinsätze fahren

\subsubsection{Berufliche Interessenvertretung außerhalb des Betriebes}

Die Engagementquoten im Bereich der beruflichen Interessenvertretung außerhalb des Betriebes haben sich in den letzten zwanzig Jahren kaum verändert. Ungefähr zwei Prozent der Menschen ab 14 Jahren in Deutschland engagieren sich in diesem Bereich; das sind sowohl im Jahr 1999 als auch 2019 jeweils rund 1,4 Millionen Menschen - sie engagieren sich beispielsweise in einer Gewerkschaft oder in einem Berufsverband. Zwischen 2004 und 2009 ist der Anteil der Engagierten von 1,9 Prozent auf 1,5 Prozent etwas gesunken und 2014 wieder auf 2,2 Prozent gestiegen (Abbildung 5-13). Im Jahr 2019 engagieren sich dann mit 1,9 Prozent etwas weniger Menschen in diesem Bereich.

Die Geschlechterdifferenzen in der beruflichen Interessenvertretung außerhalb des Betriebes sind vergleichsweise groß: Hier engagieren sich 2,5 Prozent der Männer und 1,3 Prozent der Frauen in Deutschland. Der Anteil der Engagierten ist in den mittleren Altersgruppen (30 bis 49 Jahre und 50 bis 64 Jahre) am höchsten - also bei jenen Personen, die dem Arbeitsmarkt vornehmlich zur Verfügung stehen und dementsprechend auch die Anknüpfungspunkte haben, um sich im Bereich der beruflichen Interessensvertretung zu engagieren.

Beispiele für Tätigkeiten der Engagierten im Bereich der beruflichen Interessenvertretung außerhalb des Betriebs im FWS 2019:

- Gewerkschaft: Vorstand von Arbeitskreisen

- Ortsverband einer Gewerkschaft: Kassenprüfer

- Berufsverband: stellvertretende Vorsitzende

- Ehrenamtliche Rentenberaterin: Rechtsberatung, Rechtsbetreuung

- Handwerkskammer: Vorsitzende des Prüfungsausschusses

- Seniorenvertretung der Gewerkschaft Verdi: Schriftführer, Organisation von Tagesfahrten

- Ärztekammer und kassenärztliche Vertretung: Beratertätigkeiten

- Jagdgenossenschaft: Finanzen verwalten

- Deutsche Feuerwehrgewerkschaft: bei der Organisation von Demonstrationen mitgewirkt 
- Rechtsanwaltskammer: Organisation von Veranstaltungen

- Verein für Selbstständige: Vorschläge einbringen, Weiterbildungen organisieren

Abbildung 5-13 Anteile engagierter Personen im Bereich der beruflichen Interessenvertretung außerhalb des Betriebes (Basis: alle Befragten)

Im Zeitvergleich

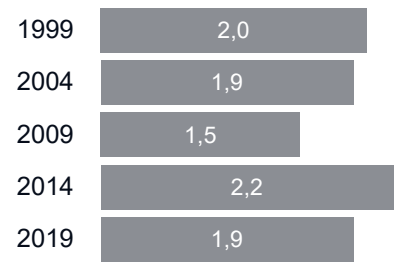

Anteile engagierter Personen 2019
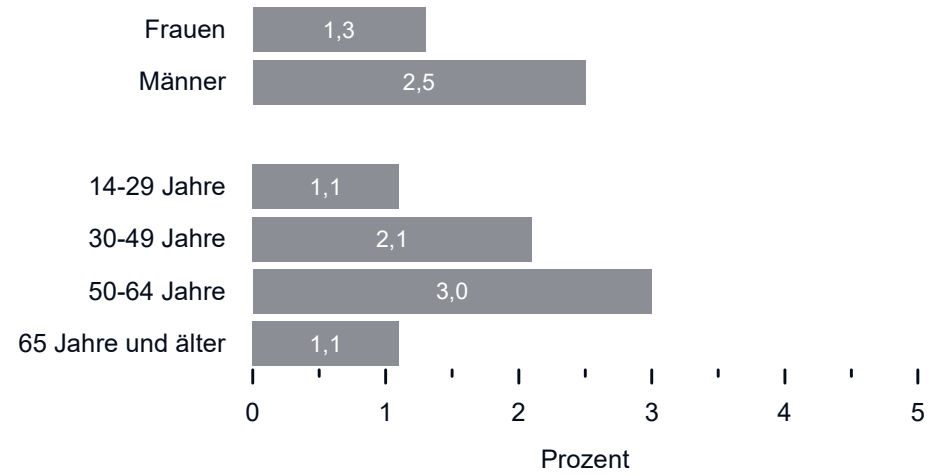

Quelle: FWS, gewichtet, eigene Berechnungen (DZA). Basis: alle Befragten. FWS 1999 ( $n=14.914$ ), FWS 2004 ( $n=15.000$ ), FWS 2009 $(n=20.003)$, FWS $2014(n=28.690)$, FWS $2019(n=27.762)$.

Statistisch signifikante Unterschiede gibt es im Zeitvergleich zwischen 2009 zu allen anderen Erhebungsjahren sowie zwischen 2014 und 2019. Der Geschlechterunterschied ist statistisch signifikant. Bei den Altersgruppen ist nur der Unterschied zwischen den 14- bis 29-Jährigen und den 65-Jährigen und Älteren nicht statistisch signifikant.

\subsubsection{Justiz und Kriminalitätsprobleme}

In dem Bereich Justiz und Kriminalitätsprobleme sind anteilig nur wenig Menschen freiwillig engagiert. Die Menschen engagieren sich hier zum Beispiel in einem Schöffenamt oder sie übernehmen die Betreuung von Straffälligen oder Verbrechensopfern. o,6 Prozent der Personen ab 14 Jahren engagieren sich 2019 in diesem Bereich. Auch 1999 waren es o,6 Prozent, das sind demnach sowohl 1999 als auch 2019 jeweils etwas mehr als 400.000 Menschen. 
Abbildung 5-14 Anteile engagierter Personen im Bereich Justiz und Kriminalitätsprobleme (Basis: alle Befragten)

Im Zeitvergleich

\begin{tabular}{|c|c|}
1999 & 0,6 \\
\hline 2004 & 0,4 \\
\hline 2009 & 0,5 \\
\hline 2014 & 0,6 \\
\hline 2019 & 0,6 \\
\hline
\end{tabular}

Anteile engagierter Personen 2019

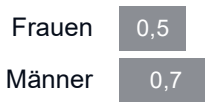

14-29 Jahre 0,1

30-49 Jahre 0,5

50-64 Jahre $\quad 1,2$

65 Jahre und älter 0,4

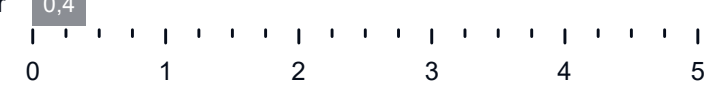

Prozent

Quelle: FWS, gewichtet, eigene Berechnungen (DZA). Basis: alle Befragten. FWS 1999 ( $n=14.916)$, FWS 2004 ( $n=15.000$ ), FWS 2009 $(n=20.005)$, FWS $2014(n=28.690)$, FWS $2019(n=27.762)$.

Es gibt keine statistisch signifikanten Unterschiede im Zeitvergleich. Der Geschlechterunterschied ist statistisch signifikant. Die Unterschiede nach Altersgruppen sind statistisch signifikant, außer der Unterschied zwischen den 30- bis 49-Jährigen und den 65-Jährigen und Älteren.

Männer sind anteilig etwas häufiger in diesem Bereich freiwillig engagiert als Frauen (Abbildung 5-14). Personen im Alter von 50 bis 64 Jahren engagieren sich im Vergleich der Altersgruppen anteilig am häufigsten (1,2 Prozent). Bei den 30- bis 49-Jährigen und den 65-Jährigen und Älteren ist der Anteil etwas geringer. Den geringsten Anteil Engagierter in diesem Bereich haben die 14- bis 29-Jährigen mit 0,1 Prozent.

Beispiele für Tätigkeiten der Engagierten im Bereich Justiz und Kriminalitätsprobleme im FWS 2019:

- Arbeitsgericht: ehrenamtlicher Richter

- Amtsgericht: Schöffin

- Senatsverwaltung: Beratung

- Weißer Ring: Opferhilfe

- Schiedsamt: Streitschlichtung für Bürger 
- Widerspruchsausschuss des Bezirksamtes: Beisitzerin

- Bewährungshelferin: gesetzliche Betreuung

- Kriminalpräventiver Rat: Auswertung von Kriminalstatistiken

- Betreuung von Jugendlichen, die zu Sozialstunden verurteilt wurden

- Präventionsarbeit mit der Polizei: Sicherheitsberater, Vorträge

\subsection{Fazit}

Die Menschen ab 14 Jahren in Deutschland üben ihr freiwilliges Engagement in sehr unterschiedlichen gesellschaftlichen Bereichen aus; die Beispiele für die Tätigkeiten der Engagierten zeigen eindrucksvoll die Vielfalt des Engagements in den einzelnen Bereichen auf. Besonders viele Menschen engagieren sich in den Bereichen Sport und Bewegung, Kultur und Musik, im sozialen Bereich oder auch im Bereich Schule und Kindergarten.

In den letzten zwanzig Jahren haben insbesondere die großen Engagementbereiche einen starken Zuwachs erfahren: Der Anteil freiwillig Engagierter ist vor allem im sozialen Bereich, in den Bereichen Kultur und Musik, Sport und Bewegung sowie Schule und Kindergarten am deutlichsten angestiegen. Auch in anderen Bereichen ist der Anteil freiwillig Engagierter von 1999 bis 2019 angestiegen, wie beispielsweise im Bereich Umwelt, Natur- und Tierschutz oder der außerschulischen Jugendarbeit und Bildungsarbeit für Erwachsene, wenn auch nicht so stark wie bei den zuvor genannten Bereichen. Gleichwohl gibt es auch Engagementbereiche, die in diesen zwanzig Jahren eine gewisse Konstanz bezüglich der Anteile an Engagierten zeigen, wie der Bereich Freizeit und Geselligkeit, die berufliche Interessenvertretung außerhalb des Betriebs, der Bereich Justiz und Kriminalitätsprobleme sowie der Bereich Unfalloder Rettungsdienst oder freiwillige Feuerwehr.

Der größte Zuwachs zwischen den letzten beiden Erhebungswellen - also zwischen 2014 und 2019 - ist für das freiwillige Engagement für die Umwelt, den Naturschutz oder den Tierschutz zu erkennen. Das verdeutlicht, dass das Engagement in diesem Bereich aufgrund der gestiegenen öffentlichen und politischen Thematisierung des Themas Klimaschutz einen Aufschwung erlebt.

In anderen Bereichen ist zwischen 2014 und 2019 wiederum ein leichter Rückgang der Engagementbeteiligung zu verzeichnen, wie beispielsweise für das Feld Sport und Bewegung. Dies stimmt mit den Ergebnissen des Sportentwicklungsberichts des Deutschen Olympischen Sportbundes (DOSB) überein, der ebenfalls einen Rückgang in den letzten Jahren verzeichnet (Breuer \& Feiler 2019: 23). Hinter diesen Entwicklungen können Herausforderungen stehen, mit denen Vereine schon seit geraumer Zeit konfrontiert sind. Menschen für - vor allem langfristige - freiwillige und ehrenamtliche Tätigkeiten zu gewinnen, stellt eine große Herausforderung dar (Breuer \& Feiler 2019). Zugleich können auch die von Abwanderung betroffenen ländlichen Räume oder der geringere Anteil junger Menschen an der Bevölkerung in 
Verbindung mit den sinkenden Zahlen freiwillig Engagierter in diesem Bereich stehen (Deutscher Bundestag 2017: 451 ff.; Priemer et al. 2019).

Traditionelle Muster der geschlechtsspezifischen Rollenzuschreibung spiegeln sich auch im Jahr 2019 in den Anteilen von Frauen und Männern in den jeweiligen Engagementbereichen wider. Frauen engagieren sich beispielsweise häufiger als Männer in Bereichen, die als familienbezogen oder sozial charakterisiert werden können, wie in der Schule und dem Kindergarten, im kirchlichen oder religiösen Bereich oder auch im sozialen Bereich. Das Engagement von Männern ist vor allem in den Bereichen Sport und Bewegung, Unfall- oder Rettungsdienste oder freiwillige Feuerwehr und auch im Bereich der Politik und der politischen Interessenvertretung höher.

Ebenso schlagen sich altersspezifische Interessen, Aufgaben sowie Gelegenheiten (Blinkert \& Klie 2017; Vogel et al. 2017) in den gesellschaftlichen Bereichen des Engagements nieder. Die Personen der jüngsten Gruppe im Alter von 14 bis 29 Jahren engagieren sich häufig im Bereich Sport und Bewegung sowie in Unfall- und Rettungsdiensten oder in der freiwilligen Feuerwehr. Hier können die eigene Freizeitgestaltung sowie auch die größere Zeitautonomie im Vergleich zu den anderen Altersgruppen eine Rolle für die Übernahme freiwilliger Tätigkeiten spielen. Zwischen 30 und 49 Jahren haben viele Menschen Kinder, die Ausgangspunkt für die Übernahme freiwilliger Tätigkeiten in der Schule, im Kindergarten oder auch in Sportvereinen sein können. Im sozialen Bereich engagieren sich vor allem die Personen im Alter von 50 bis 64 Jahren sowie die 65-Jährigen und Älteren.

Die Engagementquoten der einzelnen Bereiche spiegeln nicht nur die Interessen der freiwillig Engagierten wider, sondern beispielsweise auch, wie groß die Nachfrage nach Engagierten oder wie hoch die Anzahl an Organisationen in den jeweiligen Bereichen ist. Bei der Betrachtung und Interpretation der Ergebnisse zum freiwilligen Engagement in den verschiedenen gesellschaftlichen Bereichen ist auch zu beachten, dass sich die Befragten beim Interview mit ihrer eigenen Angabe selbst in einen der Bereiche einordnen. So könnte beispielsweise eine Tätigkeit in einem Sozialausschuss im örtlichen Stadtrat dem politischen Bereich oder dem sozialen Bereich zugeordnet werden. Unabhängig von gesellschaftlichen Gegebenheiten und persönlichen Umständen können somit auch der Schwerpunkt der freiwilligen Tätigkeit und die eigene Wahrnehmung der Befragten eine Rolle spielen.

\section{Literatur}

Alscher, M., Priller, E., \& Burkhardt, L. (2021). Zivilgesellschaftliches Engagement. In: Statistisches Bundesamt (Destatis), Wissenschaftszentrum Berlin für Sozialforschung (WZB) \& Bundesinstitut für Bevölkerungsforschung (BiB) (Hrsg.) Datenreport 2021. Ein Sozialbericht für die Bundesrepublik Deutschland (S. 399-407). Bonn: Bundeszentrale für politische Bildung.

Blinkert, B., \& Klie, T. (2017). Formen der Solidarität. In: Statistisches Bundesamt (Hrsg.) Wie die Zeit vergeht - Analysen zur Zeitverwendung in Deutschland. Beiträge zur Ergebniskonferenz 
der Zeitverwendungserhebung 2012/13 am 5./6. Oktober 2016 in Wiesbaden (S. 195-228). Wiesbaden: Statistisches Bundesamt.

Breuer, C., \& Feiler, S. (2019). Sportvereine in Deutschland: Organisationen und Personen. Sportentwicklungsbericht für Deutschland 2017/2018 - Teil 1. Bonn: Bundesinstitut für Sportwissenschaft.

Burkhardt, L., Priller, E., \& Zimmer, A. (2017). Auf der Überholspur? Frauen und freiwilliges Engagement. In: Statistisches Bundesamt (Hrsg.) Wie die Zeit vergeht - Analysen zur Zeitverwendung in Deutschland. Beiträge zur Ergebniskonferenz der Zeitverwendungserhebung 2012/13 am 5./6. Oktober 2016 in Wiesbaden (S. 179-194). Wiesbaden: Statistisches Bundesamt.

Deutscher Bundestag (2017). Zweiter Engagementbericht. Demografischer Wandel und bürgerschaftliches Engagement: Der Beitrag des Engagements zur lokalen Entwicklung und Stellungnahme der Bundesregierung (Drucksache 18/1180o). Berlin: Deutscher Bundestag.

Kausmann, C., Vogel, C., Hagen, C., \& Simonson, J. (2017). Freiwilliges Engagement von Frauen und Männern - Genderspezifische Befunde zur Vereinbarkeit von freiwilligem Engagement, Elternschaft und Erwerbstätigkeit. Berlin: Bundesministerium für Familie, Senioren, Frauen und Jugend (BMFSFJ).

Neufeind, M., Güntert, S.T., \& Wehner, T. (2015). Neue Formen der Freiwilligenarbeit. In: T. Wehner \& S. T. Güntert (Hrsg.) Psychologie der Freiwilligenarbeit. Motivation, Gestaltung und Organisation (S. 195-220). Berlin, Heidelberg: Springer.

Priemer, J., Bischoff, A., Hohendanner, C., Krebstakies, R., Rump, B., \& Schmitt, W. (2019). Organisierte Zivilgesellschaft. In: H. Krimmer (Hrsg.) Datenreport Zivilgesellschaft (S. 7-54). Wiesbaden: Springer VS.

Vogel, C., Hagen, C., Simonson, J., \& Tesch-Römer, C. (2017). Freiwilliges Engagement und öffentliche gemeinschaftliche Aktivität. In: J. Simonson, C. Vogel \& C. Tesch-Römer (Hrsg.) Freiwilliges Engagement in Deutschland - Der Deutsche Freiwilligensurvey 2014 (S. 91-151). Wiesbaden: Springer VS.

von Rosenbladt, B. (2001). Der Freiwilligensurvey 1999: Konzeption und Ergebnisse der Untersuchung. In: B. von Rosenbladt (Hrsg.) Freiwilliges Engagement in Deutschland. Ergebnisse der Repräsentativerhebung 1999 zu Ehrenamt, Freiwilligenarbeit und bürgerschaftlichem Engagement. Band 1: Gesamtbericht (S. 31-134). Stuttgart: Kohlhammer. 